\title{
POSITION AND ANTI-COLLISION SENSOR SYSTEMS FOR LARGE MANIPULATORS
}

\author{
F. Johannessen \\ F. Kristensen \\ J. Rasmussen \\ Danish Technological Institute \\ DK-2630 Taastrup, Denmark
}

\section{SUMMARY}

This paper is dealing with two sensor systems for a large manipulator with the reach of 20 meters, which are under development as a part of a running ESPRIT II project.One system is an opto-electronic position sensor system closely linked to the manipulator arm, which has the aim at increasing accuracy and speed. The other system is an ultrasound anti-collision sensor system capable of detecting unexpected obstacles within the manipulator's workspace to increase safety of operation.

\section{INTRODUCTION}

Industrial robots have been utilized for many years and are now highly sophisticated and automated. An important technique, which has contributed to this - especially within the last few years - is sensor systems, which make it possible to determine parameters of the robot as well as its surroundings during operation. However, special problems affect the operation of large manipulators and raise the need for new sensor systems.

This presentation is about the sensor development for a highly automated robot with very large reach, suited to work in hostile environments, which makes it interesting also within the construction industry.

Fig. 1 shows a large manipulator developed in Germany, which forms the basis of the development of a new one, which is mechanically very similar, but has a higher degree of automation and accuracy. The development is performed within the ESPRIT II programme by an international consortium under the project name LAMA (LArge MAnipulator), project No.EP2280. The target machine is planned to reach the following specifications:

* $\quad 20 \mathrm{~m}$. reach with 5 links

* $\quad 0,5 \mathrm{~m} / \mathrm{sec}$. max speed

* $\quad 400 \mathrm{~kg}$. pay load

* $\quad \pm 10 \mathrm{~mm}$. precision

* Vibration damping

The actuators are hydraulic, and each actuator has a local servo control. Sensor signals and control commands are transmitted via an optical fibre line to the central robot controller. 
The tasks of sensors are:

1. To determine on-line the position of the Tool Center Point, TCP, taking into account that the TCP deviates up to half a meter due to deflections caused by load and accelerations, and also that direct sight is not possible due to obstacles in the work space.

2. To supply signals necessary for the active damping of oscillatory movements. This will increase maximum speed and acceleration.

3. To improve safety by detecting:

- $\quad$ fixed objects in the work space (objects not registered in the central controller)

- $\quad$ objects moving into the work space

- $\quad$ human beings moving into the work space

In Fig. 2 is schematically shown how both signals are connected to the transmission-line (SERCOS), and also that the position-signal is used for damping the vibrations in the arm caused by heavy loads and accelerations.

\section{POSITION MEASUREMENT}

The traditional way of measuring the position in space of a manipulator's TCP is by mounting an angle measuring device on each joint, e.g. a resolver, an optical angle encoder or possibly a linear position sensor mounted on the hydraulic cylinder. However, the signals from these sensors are for many purposes insufficient for calculation of the TCP position with the desired accuracy, due to the inevitable deformations within these large structures, which cannot be regarded as perfectly stiff.

In Fig. 3 is schematically shown a proposal for a system, which is under development at the Danish Technological Institute, which highly improves resulting accuracy. On each joint is mounted an opto-electronic device (symbolized with coordinate systems), which measures the position and orientation of the next device, forming a chain-like structure starting on the ground under the manipulator and ending up at the TCP.

Fig. 3 (center) shows an example on how this measurement can be performed (other configurations are possible). An angle encoder or resolver measures the angle between the two links and non-tactile opto-electronic devices measure the small deviations from the theoretical positions given by the encoder signal. This way of splitting the measurement is attractive, because the opto-electronic devices can be made fairly simple and reliable, and chiefly be made from standard components, so that no "high-tech" solution is required, but unlike encoders, these devices normally only work within a small angular range.

Theoretically, six parameters have to be measured to define the relative position and orientation of a device. These could be a distance, two translational deflections, two angular deflections and a torsional deflection. Great difficulties could be involved with measuring the distance, but fortunately this can normally be considered as constant, so the measurement of this parameter is omitted. In some cases also the torsional deflection is of minor importance, e.g. if the transverse forces and accelerations are small, but most likely this situation will not occur in practice. 


\section{TRANSLATIONAL AND ANGULAR DEFLECTION}

Fig. 4 shows how the translational and angular deflections can be measured by imaging two light sources (infrared LEDs) via a lens onto a two dimensional position sensitive detector, both directly and via a plane mirror, which converts the angular deflection into a deflection of a light spot on the sensor element. The sensitivity and range for these measurements can simply be adjusted by changing the position and the focal length of the lens. Different sensor elements are available, such as area scan CCD sensors, which have a high linearity, or analogue sensors, which are simple in operation and very fast (these have been chosen for our experiments). Linear analogue sensors could also be a relevant choice due to their low price, but in this case a more complex optical system will be needed.

\section{TORSIONAL DEFLECTION}

In Fig. 5 is shown a way of measuring the torsional deflection by emitting linearly polarized light and at the other end to measure the change in polarization angle by a specially designed sensor system. This can be configured in different ways: Here is shown an example where beam-splitters (polarizing or equipped with polarization filters) is dividing the incident beam of polarized light into two, which then hit two photo diodes. When the polarization angle changes, signal from one diode increases and the other decreases in a way, so that the ratio between difference and sum will become a function of the angle, in principle independent of amplitude as well as geometrical inhomogenities of the incident light distribution.

This torsion sensor has for our purpose an unnecessary large range in measuring angle and accordingly a similarly low sensitivity, but this can not be altered optically as in the previous case. To reach the goal for the accuracy, which for large manipulators preferably is better than approximately 2 minutes of arc, it is necessary that the two signal paths created by the beamsplitter are kept geometrically, thermally and electrically identical to as high an extend as pos sible. Furthermore, the beam-splitter should be of a type, where the splitting-ratio is independent of the angle of incidence within the small range, defined by the actual range for the translational deflection, otherwise the torsion signal will be influenced by the translational deflection.

To obtain the best signal to noise ratio and to avoid sources of error such as stray light, sensor dark current and amplifier off-set, the emitted light is pulsed and the received signals detected by lock-in amplifiers. This is also the case for the signals from the analogue position sensitive detectors.

\section{STATE OF DEVELOPMENT}

Experiments with the essential parts of the sensor system have taken place, and they have been very successful in reaching the goals for precision and speed. The development of the entire system including mechanics for mounting, angle encoder, signal conditioner and interface is now taking place.

An important problem to be dealt with is the environmental protection. When this optical measurement system has to work in a harsh environment, industrial or outdoors, it must be protected by an air tight encapsulation. This is even the case for the light path stretching from 
joint to joint, as contamination of windows with e.g. dust or precipitation may disturb the measurement. The cost of this encapsulation is expected to form a considerable part of the total cost for the measuring system.

\section{ANTI-COLLISION}

The aim of this development is to detect objects within a distance of up to app. 3 meters from the links as indicated in Fig. 3. Objects detected will be compared with objects registered in the central controller memory, so that the manipulator can be stopped, if there is a collision risk.

Ultrasound has been chosen as detection principle for the following reasons:

- The aim at application in hostile environment excludes optics.

- The presence of large iron masses make electromagnetic methods difficult.

- $\quad$ Price seems relative favourable.

\section{THE ULTRA SOUND SYSTEM}

The system works by measuring echoes received from obstacles within the close vicinity of the manipulator arm. The ultrasound transducers chosen for our experiments operate as both emitters and sensors at a frequency of app. $40 \mathrm{kHz}$. They have a directivity of app. $100^{\circ}$, which means that they cover a very wide angle in front of them. These transducers, together with the associated electronics, are mounted with certain intervals all the way along the manipulator arm. Together they establish a warning zone, which envelopes the manipulator. A reflecting object within this zone will cause the sensor system to send a warning signal to the central computer.

Fig. 3 (bottom) indicates how transducers will be controlled: Half of the transducers (marked A) emit ultrasonic bursts and all transducers listen, then there is a shift to the other half (marked B), and so on. The time of flight for an ultrasound burst travelling from the emitting transducers and back to itself or one of its nearest neighbours is used to define the warning zone, which is thereby limited by a system of spheres and ellipsoides. As no high accuracy measurement is needed, the variations in the propagational speed of sound can be neglected, a calibration would however be preferable.

In most ultrasound ranging applications a very narrow beam is utilized. This is not the case for this system, where a wide angle of sensitivity is needed to cover the surroundings of the manipulator arm. This large angle results in very weak signals, and problems are also caused by the demands for detecting the most various surfaces.

Each transducer and its electronics for emitting as well as receiving is mounted in one block as shown on Fig. 6. For two reasons, the frequency of the ultrasound bursts is swept within a small range: It compensates for the small variations in resonance frequency of the individual transducers, and extinction of an echo due to interference is avoided.

In order to avoid the manipulator itself from beeing detected, some of the transducers must be closed dowm in accordance with the angle between the links as indicated on Fig. 7. 
The ultrasound transducers are controlled by an anti-collision controller fitted to each link of the manipulator. The close down of individual transducers and their timing, as well as communication with the central controller is performed by this unit. The communication contains warning signals including adresses showing which unit they come from.

\section{EXPERIMENTS}

Preliminary experiments with a small scale system have shown good detection performance for distances up to 2 meters. The reflecting objects have been hard objetcs with dimensions down to about $10 \mathrm{~cm}$, as well as human beeings. An improved version of the electronics is under development and is expected to give an increase in maximum distance. However, any drastic improvements are not expected due to the fact, that the intensity of the echo drops rapidly, when the distance transducer-object increases.

For the comparison of different sensor systems a few standard objects have been chosen (a plane cirkular plate, a tube and a cube). When it comes to specifying the sensitivity of the anticollision sensor system, these objects are unfortunately insufficient, and a great number of experiments have to be performed. This because there is an innumerable number of different objects to take into account, and the intensity of reflection will depend on many parameters: material, shape, size, orientation, etc.

\section{CONCLUSION}

Two new sensor systems for manipulators with very large reach have been described. In laboratory tests they have shown a promising performance, and we are convinced that they will open up new areas of application for large manipulators due to their improvement of accuracy and safety.

In addition it is our hope that these sensor systems can prove useful within related areas concerning large structures.

\section{REFERENCES}

1. A. Gugg-Helminger, W. Dähn:

Grundlagen der optischen Koordinatenmessgeräte. Opto Elektronik Magazin Vol. 3, no. 2, 1987.

2. L. Kay:

Airborne ultrasonic imaging of a robot work space. Sensor Review Jan. 1985. 


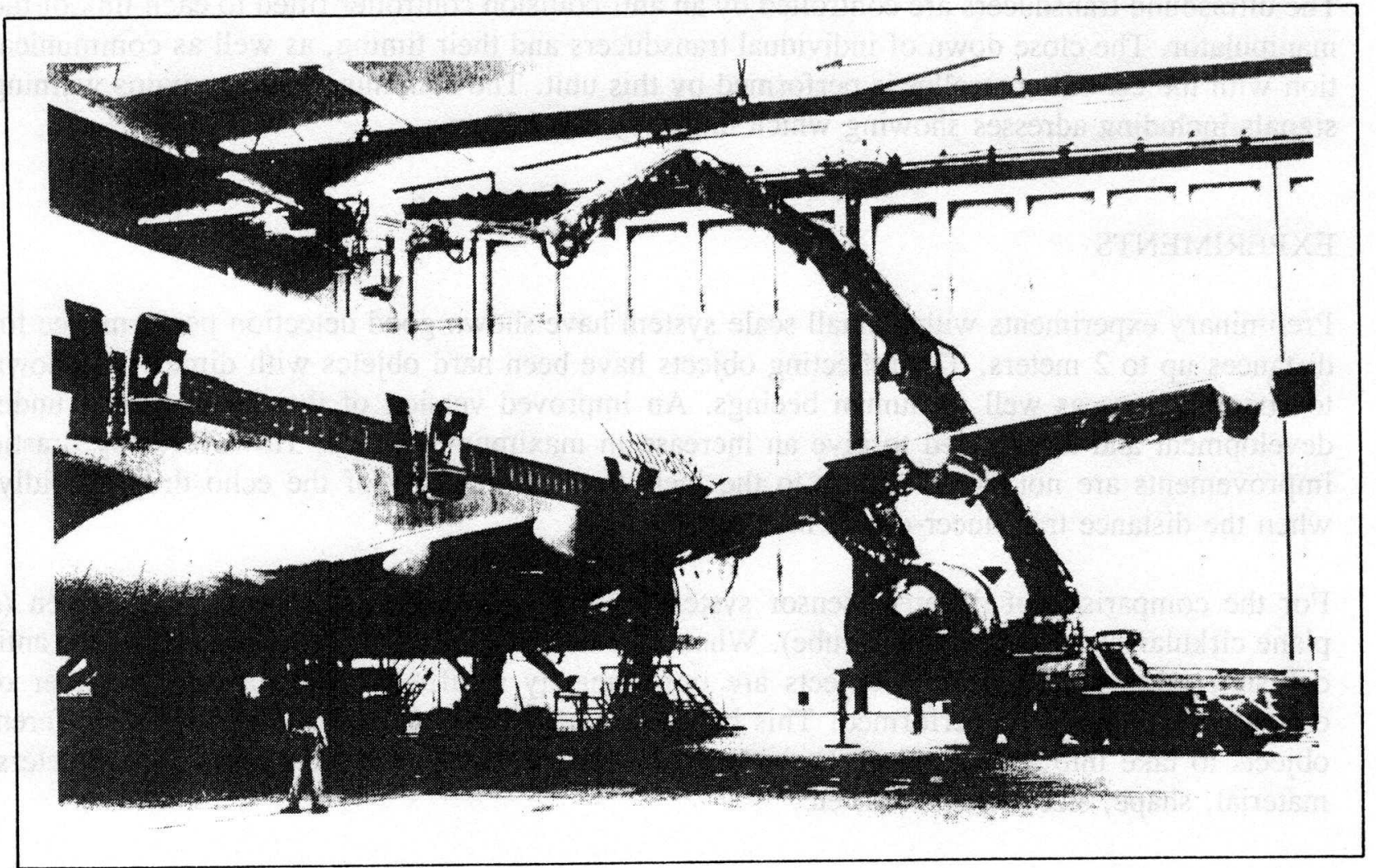

Fig. 1

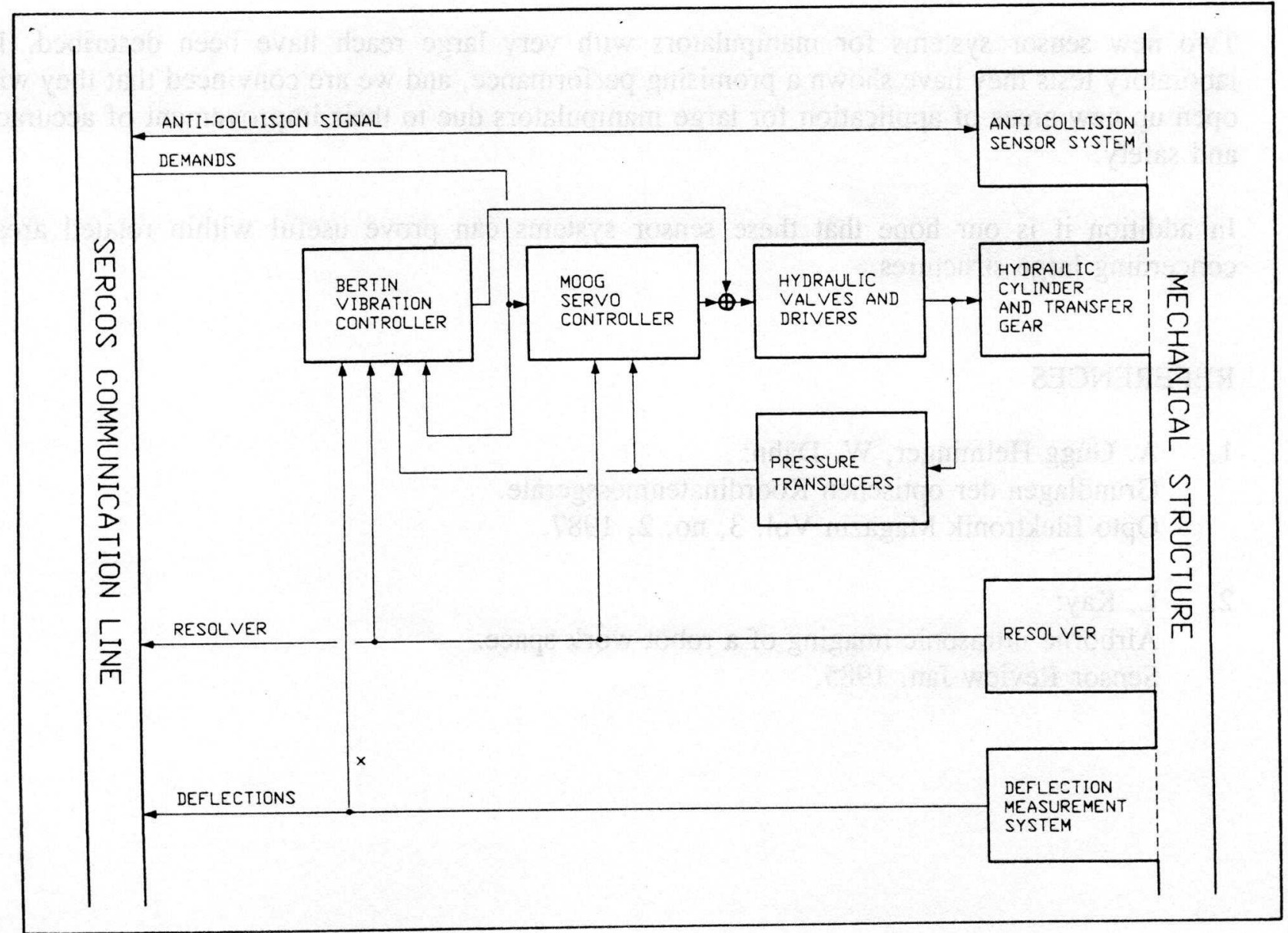

Fig. 2 


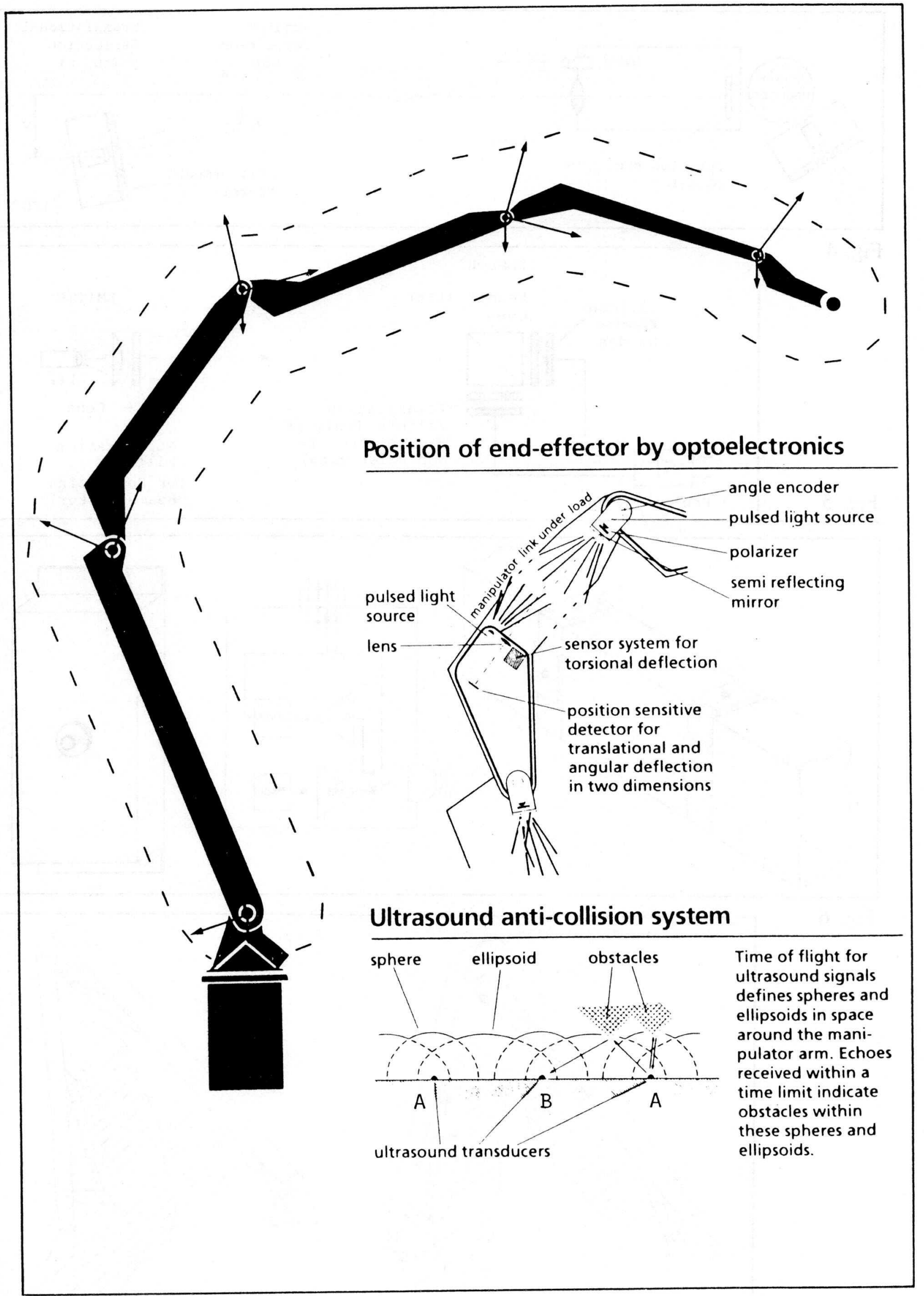

Fig. 3 


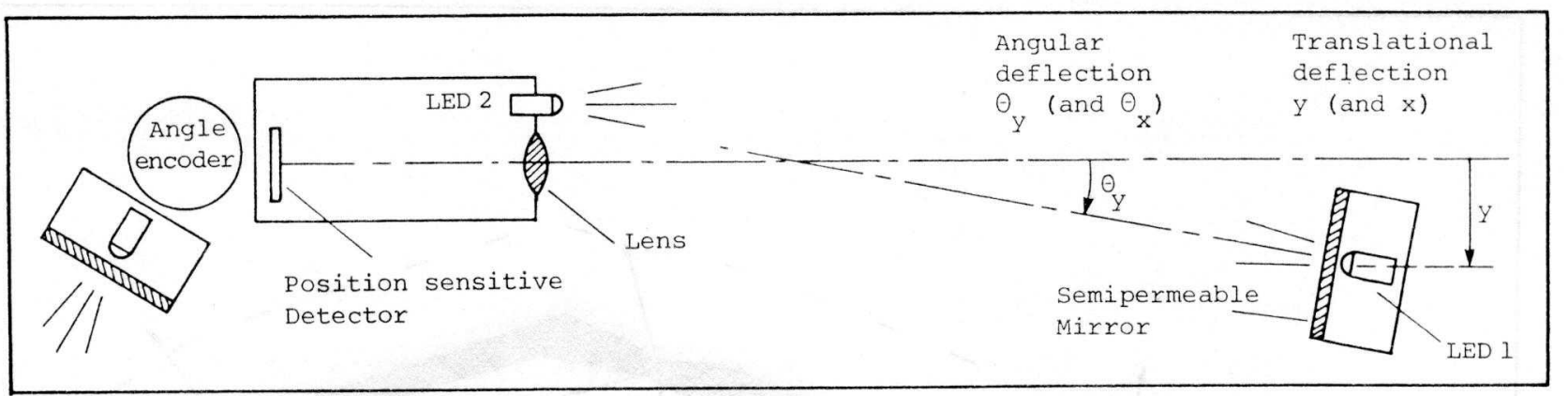

Fig. 4

Fig. 5
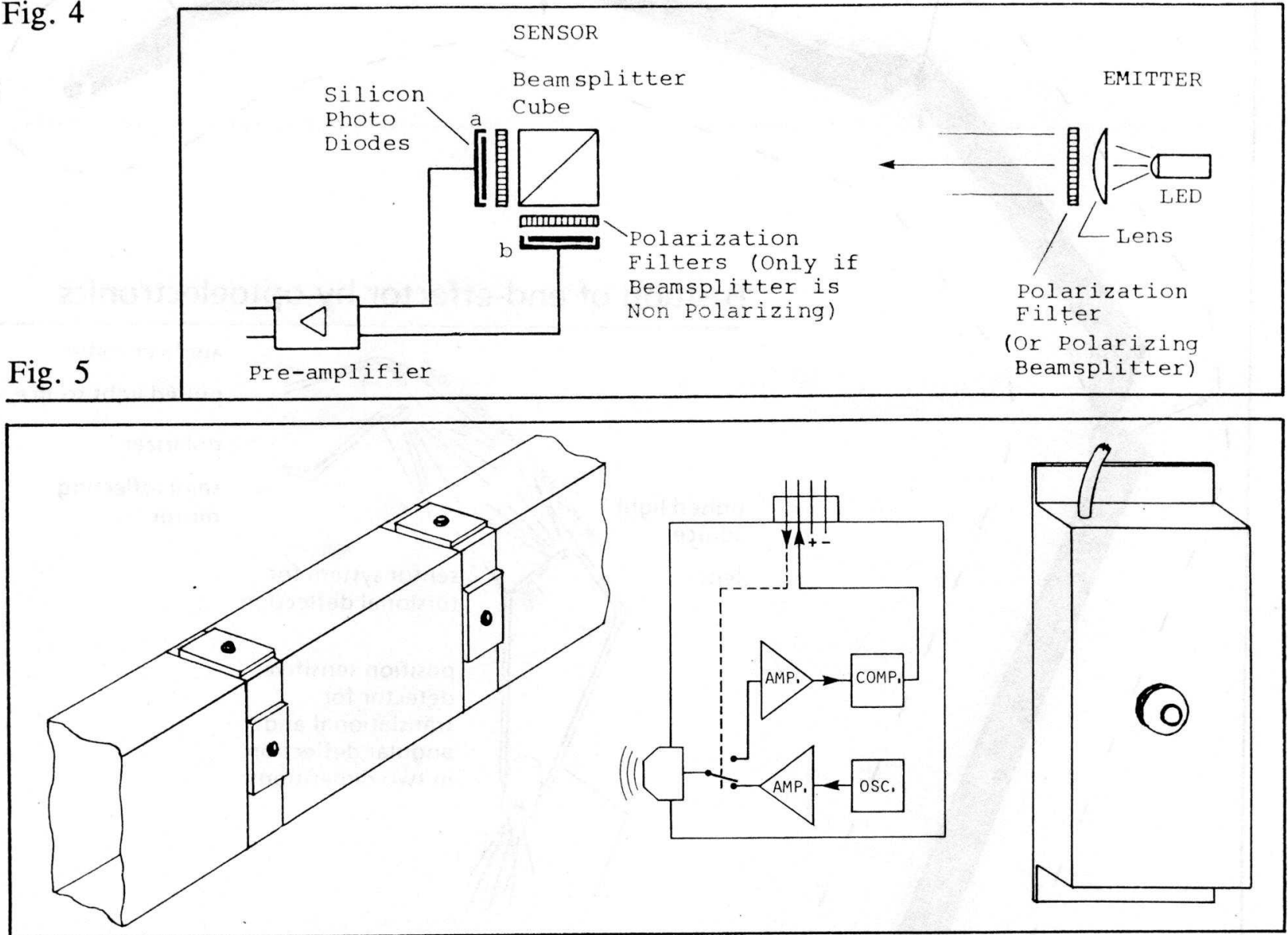

Fig. 6

Fig. 7
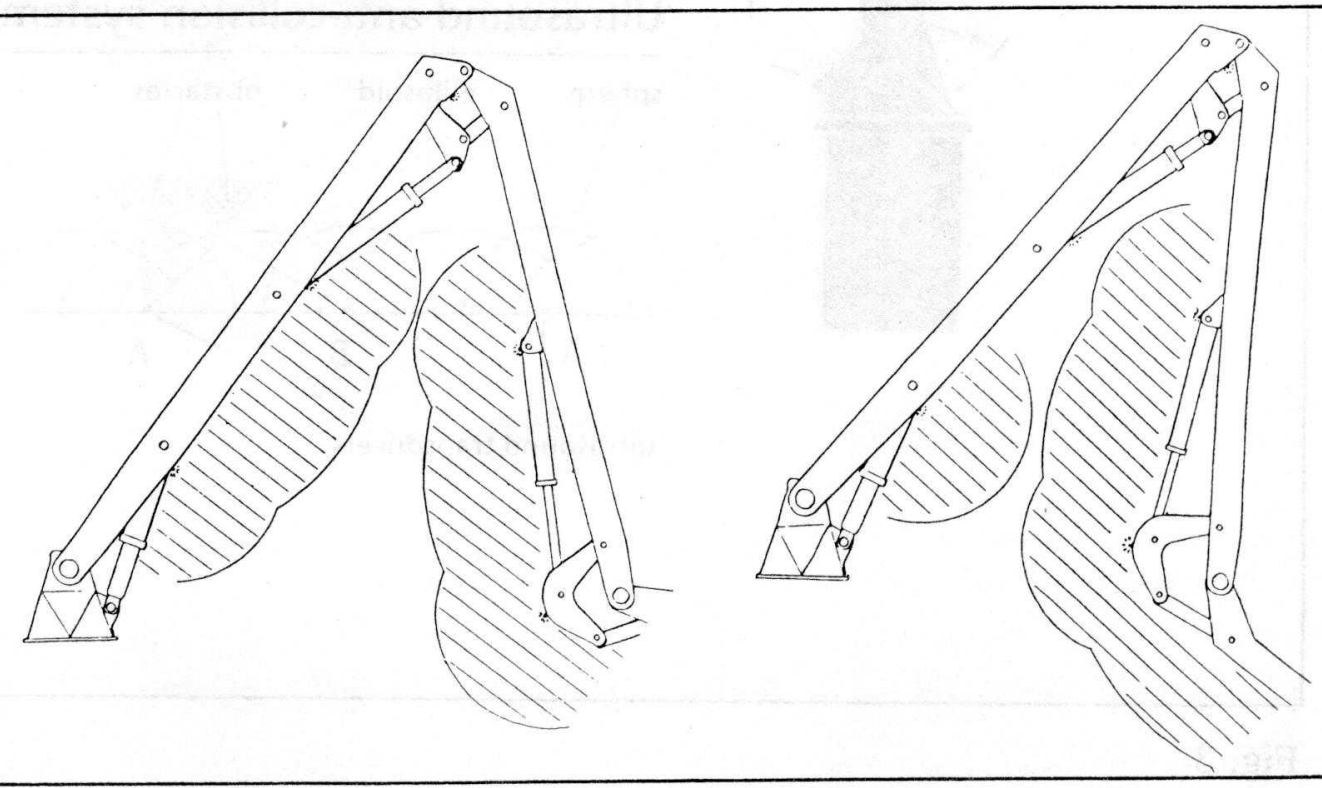